\title{
An approach to the young hypertensive patient
}

\author{
P Mangena, ${ }^{1}$ MB ChB, FCP (SA); S Saban, ${ }^{2} \mathrm{MB}$ ChB, MFamMed, FCFP (SA); \\ K E Hlabyago, ${ }^{3}$ BSc (Education), MSc, MB ChB, MMed (Family Medicine); B Rayner, ${ }^{1}$ MB ChB, MMed, FCP (SA), PhD
}

${ }^{1}$ Division of Nephrology and Hypertension, Faculty of Health Sciences, Groote Schuur Hospital and University of Cape Town, South Africa

${ }^{2}$ Private Practice, and Division of Family Medicine, School of Public Health and Family Medicine, Faculty of Health Sciences,

University of Cape Town, South Africa

${ }^{3}$ Department of Family Medicine, Dr George Mukhari Academic Hospital and Sefako Makgatho Health Sciences University, Pretoria, South Africa

Corresponding author: P Mangena (mngphe002@myuct.ac.za)

Hypertension is the leading cause of death worldwide. Globally and locally there has been an increase in hypertension in children, adolescents and young adults $<40$ years of age. In South Africa, the first decade of the millennium saw a doubling of the prevalence rate among adolescents and young adults aged 15 - 24 years. This increase suggests that an explosion of cerebrovascular disease, cardiovascular disease and chronic kidney disease can be expected in the forthcoming decades. A large part of the increased prevalence can be attributed to lifestyle factors such as diet and physical inactivity, which lead to overweight and obesity. The majority $(>90 \%)$ of young patients will have essential or primary hypertension, while only a minority $(<10 \%)$ will have secondary hypertension. We do not recommend an extensive workup for all newly diagnosed young hypertensives, as has been the practice in the past. We propose a rational approach that comprises a history to identify risk factors, an examination that establishes the presence of target-organ damage and identifies clues suggesting secondary hypertension, and a limited set of basic investigations. More specialised tests should be performed only where there is a clinical suspicion that a secondary cause for hypertension exists. There have been no randomised clinical trials on the treatment of hypertension in young patients. Expert opinion advises an initial emphasis on lifestyle modification. This can comprise a diet with reduced salt and refined carbohydrate intake, an exercise programme and management of substance abuse issues. Failure of lifestyle measures or the presence of target-organ damage should prompt the clinician to initiate pharmacotherapy. We recommend referral to a specialist practitioner in cases of resistant hypertension, where there is severe target-organ damage and when a secondary cause is suspected.

S Afr Med J 2016;106(1):36-38. DOI:10.7196/SAMJ.2016.v106i1.10329 


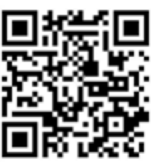

Hypertension is a major cause of mortality and morbidity globally. It is estimated that $>1$ billion persons worldwide suffer from hypertension..$^{[1]}$ In the 2010 Global Burden of Disease study ${ }^{[2]} 9$ million deaths were attributed to hypertension, making it the leading cause of death worldwide.

The rate of hypertension has risen over the past few decades to reach epidemic proportions among the young ${ }^{[3]}$ (defined in this article primarily as adolescents and adults $<30$ years of age). There is a paucity of data on the best way to treat younger patients, and most recommendations are extrapolated from evidence in older patients. ${ }^{[4]}$

This rapid rise is of concern, as it portends an explosion of cardiovascular disease, cerebrovascular disease and chronic kidney disease in the coming years. Furthermore, due to the traditional approach that extensively investigated young hypertensives in search of a secondary cause, there are high healthcare costs associated with this epidemic.

This article provides a brief overview of hypertension in South African (SA) adolescents and young adults. Specific reference is made to diagnostic criteria, treatment considerations and referral options.

\section{Epidemiology}

Hypertension is defined as a persistent elevation of office blood pressure $>140 / 90 \mathrm{mmHg}$. Approximately a third of the adult SA population is hypertensive. ${ }^{[5]}$ Among adolescents and young adults (15 - 24 years old) the incidence is $\sim 10 \% .{ }^{[5]}$ Worryingly, there has been a startling increase, with the prevalence approximately doubling in this age group within a decade. ${ }^{[3]}$ The reasons include the 'risk transition', which is characterised by urbanisation, a change in diet and lower levels of physical activity. ${ }^{[6]}$

There are few data on the long-term outcomes of hypertension in the young. However, the available evidence shows that young people with hypertension have similar target-organ damage as older hypertensives, such as left ventricular hypertrophy (LVH), microalbuminuria and carotid intimal thickness. These are associated with adverse cardiovascular outcomes. ${ }^{[7,8]}$

\section{Pathophysiology}

The causes of hypertension in the young can be divided into primary and secondary.

\section{Primary hypertension}

Primary hypertension (also called essential hypertension) has no specific cause, although genetic and environmental factors play an important role. ${ }^{[9]}$ More than $90 \%$ of young people with hypertension have primary hypertension. ${ }^{[7,10]}$ It is often associated with a family history of hypertension and frequently accompanied by obesity or the metabolic syndrome. International population surveys show an increase in obesity in children and adolescents, which parallels an increase in the prevalence of hypertension in the same age groups. ${ }^{[11]}$ Hypertensive children and adolescents go on to become hypertensive adults - a phenomenon known as 'BP tracking. ${ }^{[12]}$

Novel mechanisms implicated in the pathogenesis include low birth weight (the Barker-Brenner hypothesis) ${ }^{[13]}$ and hyperuricaemia. ${ }^{[14,15]}$ Low-birth-weight and/or small-for-gestational-age infants have a lower nephron number at birth (the so-called 'nephron endowment'), which induces physiological changes that lead to hypertension and chronic kidney disease later in life. ${ }^{[13]}$ Hyperuricaemia as a cause for hypertension in adolescents is an intriguing concept. Experimental data indicate that hyperuricaemia invokes renal changes that drive hypertension. This 'hyperuricaemic hypertension' has in small studies been shown to be reversible with urate-lowering therapy, at least in the early stages, i.e. soon after diagnosis. (There is not yet enough evidence to recommend routine use of urate-lowering therapy in young patients..$^{[14,15]}$

\section{Secondary hypertension}

This form of hypertension affects approximately $10 \%$ of young hypertensives. ${ }^{[7]}$ The probability of secondary hypertension is inversely proportional to the age of the patient (i.e. higher in a school-going child, but lower in a young adult). ${ }^{[7]}$ The importance of identifying secondary hypertension lies in the potential for cure with appropriate treatment.

The causes of secondary hypertension can be further divided as follows:

- renal parenchymal disease (e.g. glomerulonephritis)

- renovascular disease (e.g. renal artery stenosis)

- mineralocorticoid-mediated hypertension (e.g. primary hyperaldosteronism)

- catecholamine-mediated hypertension (e.g. phaeochromocytomas)

- medication (e.g. the oral contraceptive pill)

- abuse of cocaine or amphetamines ${ }^{[16]}$

- coarctation of the aorta

- rarer causes.

\section{Clinical features}

Most young patients are asymptomatic and diagnosed during screening or when presenting with an unrelated condition. It is therefore imperative that opportunistic screening should take place at every opportunity. A minority present with a hypertensive emergency (such as heart failure, renal failure or malignant hypertension).

The history should enquire about whether a raised blood pressure has been recorded previously and the circumstances in which the hypertension was first noted; diabetes mellitus and premature cardiovascular disease; use of medication (especially oral contraceptives); use of alcohol and tobacco products; and abuse of illicit drugs, especially methamphetamines (commonly known as 'tik'), which is associated with severe hypertension and chronic kidney disease. ${ }^{[17]}$

Blood pressure should be recorded using an approved and calibrated electronic device or mercury sphygmomanometer. Raised blood pressure should be confirmed with multiple readings, and consideration should be given to performing out-of-office blood pressure measurement (such as home blood pressure or 24-hour ambulatory blood pressure monitoring) to exclude 'white coat' hypertension. ${ }^{[4]}$

The examination is focused on establishing if there is target-organ damage or whether there are features that suggest a secondary cause. The presence of target-organ damage should prompt the institution of pharmacotherapy and closer follow-up. ${ }^{[4]}$ The clinician should look for:

- an elevated body mass index (BMI) and increased waist circumference

- features of insulin resistance (e.g. acanthosis nigricans)

- urinary dipsticks to detect primary renal disease (e.g. glomerulonephritis) or renal damage

- a pressure overloaded apex in keeping with LVH

- fundoscopy to check for retinal hypertensive changes.

Features that point to a secondary cause include:

- pulse discrepancies (this may suggest a vasculopathy, such as Takayasu's arteritis)

- radiofemoral delay (this suggests coarctation of the aorta)

- abdominal bruits (these are suggestive of renal artery stenosis) 
- flushing, sweating and tachycardia (this may be a clue to the presence of a phaeochromocytoma)

- acne, striae, moon facies (these suggest Cushing's syndrome)

- positive dipsticks for blood and/or protein.

\section{Investigations}

We recommend that young hypertensives are not routinely investigated for secondary causes, given the scale of the epidemic and that the majority of patients have primary hypertension. Baseline tests that should be routinely performed include: ${ }^{[4]}$

- serum potassium (hypokalaemia may suggest primary hyperaldosteronism)

- serum creatinine and determination of estimated glomerular filtration rate

- urine microalbumin-to-creatinine ratio

- fasting blood glucose and lipogram

- 12-lead electrocardiogram (ECG). The clinician should note that the ECG is not validated for diagnosis of LVH in individuals $<30$ years, and should be interpreted with caution. ${ }^{[16]}$

- Where resources allow, an echocardiogram to evaluate for LVH may be performed, which is the gold standard for diagnosis.

Specific tests for a secondary cause should only be performed if there is a clinical suspicion. These include:

- plasma renin and aldosterone for suspected primary hyperaldosteronism

- urinary catecholamines for suspected phaeochromocytoma

- renal sonography for suspected renal disease

- computed tomography angiography of the renal vessels for suspected renal artery stenosis

- urine screening for amphetamines and cocaine.

\section{Therapy}

Therapy for young hypertensives has not been defined by randomised clinical trials and is based on expert opinion. In all instances the main focus should be on instituting lifestyle changes. ${ }^{[4]}$ All patients should be encouraged to lose weight by eliminating refined carbohydrate from their diet, reducing saturated fat intake and undertaking an exercise programme or joining an organised sports programme. Fresh fruit and vegetables in the diet should be encouraged and salt intake must be reduced. Avoidance of junk food is strongly recommended. Alcohol use needs to be moderated and all tobacco product use discontinued. If substance abuse is detected, the patient should be referred for appropriate treatment.

The role of pharmacotherapy has not been established in young people. It should be considered in the following situations: after failure of lifestyle therapy, in patients with target-organ damage or secondary causes, and in cases of severe hypertension.

Few data are available on the choice of antihypertensive agents. It is recommended that medication should be similar to that used in adults, as recommended by the South African Hypertension Society Practice Guideline on the management of hypertension. ${ }^{[18]}$ The three front-line classes of antihypertensive agents are thiazide or thiazide-like diuretics, calcium channel blockers, and either an angiotensin-converting enzyme (ACE) inhibitor or angiotensin receptor blocker. However, it is recommended to reserve the use of diuretics in cases of more severe hypertension or where there is a compelling indication, such as fluid overload, to avoid the long-term metabolic consequences of diuretics, especially the predisposition to diabetes mellitus. ${ }^{[4]}$

\section{When to refer}

It is no longer recommended that all young hypertensives be referred for specialist evaluation, especially if they are $>25$ years of age. Referral should be considered in the following circumstances:

- blood pressure $>180 / 110 \mathrm{mmHg}$

- the presence of target-organ damage or complications of hypertension (e.g. heart failure and renal impairment)

- resistant hypertension

- suspected secondary causes.

\section{Conclusion}

Hypertension in young people has doubled during the past 10 years and has necessitated a re-evaluation of our approach to their evaluation, investigation and treatment. There is an urgent need to address lifestyle issues that are driving this epidemic. It is also no longer recommended that all young hypertensives undergo extensive investigation or referral to a specialist. Practical recommendations regarding the investigation and treatment of young hypertensives are presented.

\section{References}

1. Alwan A. Global Status Report on Noncommunicable Diseases 2010. Geneva: World Health Organization, 2011.

2. Lim S, Vos T, Flaxman A, et al. A comparative risk assessment of burden of disease and injury attributable to 67 risk factors and risk factor clusters in 21 regions, 1990 - 2010: A systematic analysis for the Global Burden of Disease Study 2010. Lancet 2012;380(9859):2224-2260.

3. Bradshaw D, Steyn K, Levitt N, Nojilana B. Non-communicable Diseases: A Race Against Time. Cape Town: Medical Research Council South Africa, 2011.

Town: Medical Research Council South Africa, 2011.
4. Falkner B, Daniels S. Summary of the Fourth Report on the Diagnosis, Evaluation, and Treatment of Falkner B, Daniels S. Summary of the Fourth Report on the Diagnosis, Evaluation, an
High Blood Pressure in Children and Adolescents. Hypertension 2004;44(4):387-388.

5. Shisana O, Labadarios D, Rehle T, et al. South African National Health and Nutrition Examination Survey (SANHANES-1): 2014. Cape Town: HSRC Press, 2014

6. Reddy S, Resnicow $\mathrm{K}$, James $\mathrm{S}$, et al. Rapid increases in overweight and obesity among South African adolescents: Comparison of data from the South African National Youth Risk Behaviour Survey in 2002 and 2008. Am J Public Health 2012;102(2):262-268. [http://dx.doi.org/10.2105/AJPH.2011.300222]

7. Assadi F. The growing epidemic of hypertension among children and adolescents: A challenging road ahead. Pediatr Cardiol 2012;33(7):1013-1020. [http://dx.doi.org/10.1007/s00246-012-0333-5]

8. Drukteinis J, Roman M, Fabsitz R, et al. Cardiac and systemic hemodynamic characteristics of hypertension and prehypertension in adolescents and young adults: The Strong Heart Study. Circulation 2006;115(2):221-227.

9. Weber M, Schiffrin E, White W, et al. Clinical Practice Guidelines for the Management of Hypertension in the Community. A Statement by the American Society of Hypertension and the International Society of Hypertension. J Hypertens 2014;32(1):3-15.

10. Flynn JT. Hypertension in children. In: Kaplan N, ed. Kaplan's Clinical Hypertension. 9th ed. Flynn JT. Hypertension in children. In: Kaplan
Philadelphia: Lippincott Williams and Wilkins, 2006.

Philadelphia: Lippincott Williams and Wilkins, 2006.
1. Din-Dzietham R, Liu Y, Bielo M, Shamsa F. High blood pressure trends in children and adolescents in Din-Dzietham R, Liu Y, Bielo M, Shamsa F. High blood pressure tren
national surveys, 1963 to 2002. Circulation 2007;116(13):1488-1496.

12. Tirosh A, Afek A, Rudich A, et al. Progression of normotensive adolescents to hypertensive adults: A study of 26980 teenagers. Hypertension 2010;56(2):203-209. [http://dx.doi.org/10.1161/ HYPERTENSIONAHA.109.146415

3. Luyckx V, Brenner B. Birth weight, malnutrition and kidney-associated outcomes - a global concern. Nat Rev Nephrol 2015;11(3):135-149. [http://dx.doi.org/10.1038/nrneph.2014.251]

14. Feig D, Soletsky B, Johnson R. Effect of allopurinol on blood pressure of adolescents with newly diagnosed essential hypertension. JAMA 2008;300(8):924. [http://dx.doi.org/10.1001/jama.300.8.924]

5. Gaffo A, Jacobs D, Sijtsma F, Lewis C, Mikuls T, Saag K. Serum urate association with hypertension in young adults: Analysis from the Coronary Artery Risk Development in Young Adults cohort. Ann Rheum Dis 2012;72(8):1321-1327. [http://dx.doi.org/10.1136/annrheumdis-2012-201916]

16. Hancock E, Deal B, Mirvis D, Okin P, Kligfield P, Gettes L. AHA/ACCF/HRS Recommendations for Hancock E, Deal B, Mirvis D, Okin P, Kligfield P, Gettes L. AHA/ACCF/HRS Recommendations for
the Standardization and Interpretation of the Electrocardiogram: Part V: Electrocardiogram Changes the Standardization and Interpretation of the Electrocardiogram: Part V: Electrocardiogr
Associated With Cardiac Chamber Hypertrophy. Circulation 2009;119(10):e251-e261.

Associated With Cardiac Chamber Hypertrophy. Circulation 2009;119(10):e251-e261.
17. Jones E, Rayner B. Hypertension, end-stage renal disease and mesangiocapillary glomerulonephritis Jones E, Rayner B. Hypertension, end-stage renal disease and mesangiocapillary glomerulonephritis
in methamphetamine users. S Afr Med J 2015;105(3):199-201. [http://dx.doi.org/10.7196/samj.8731] 18. Seedat $Y$, Rayner B, Veriava Y. South African hypertension practice guideline 2014: Review article. Cardiovasc J Afr 2014;25(6):288-294. [http://dx.doi.org/10.5830/CVJA-2014-062] 